Peter Kupka, Joachim Möller*, Philipp Ramos Lobato und Joachim Wolff (IAB)

\title{
Teilhabe für arbeitsmarktferne Arbeitslose durch einen Sozialen Arbeitsmarkt - Chancen und Risiken eines notwendigen Instruments
}

https://doi.org/10.1515/zfwp-2018-0014

\begin{abstract}
We discuss a new scheme of subsidized jobs for unemployed people with extremely low job finding prospects. This scheme, referred to as Social Labour Market, primarily intends to promote their social inclusion. Research on public employment schemes has shown that it is of utmost importance to define very narrow criteria for participation in order to avoid lock-in-effects. Due to health issues and other severe employment impediments of the participants, the design of a social labour market should allow for flexible work arrangements and include some kind of mentoring ("job coaches"). We conclude that the scheme planned by the German government basically meets many criteria recommended by labour market researchers but still bears some risk of "creaming”. We recommend to start with a small number of participants. This should help to test the process of assigning participants and assess the effectiveness of different designs of the scheme before boosting the number of participants to the ultimate size.
\end{abstract}

\section{Einleitung}

Mit Blick auf die meist hoffnungslose Lage besonders arbeitsmarktferner Langzeitarbeitsloser wird bereits seit Einführung der Grundsicherung für Arbeitsuchende über die Schaffung eines Sozialen Arbeitsmarkts diskutiert. Gemeinhin bezeichnet dieser Begriff ein Förderinstrument, das sich auf den längerfristigen Einsatz öffentlich geförderter Beschäftigung stützt und auf diesem Wege auch jenen Leistungsberechtigten eine Beschäftigungsperspektive eröffnen soll, die aufgrund gesundheitlicher Probleme, fehlender Qualifikation oder sonstiger gravierender Einschränkungen ihrer Arbeits- und Leistungsfähigkeit keine realistische Chance

*Kontakt: Joachim Möller, Institut für Arbeitsmarkt- und Berufsforschung (IAB), Regensburger Straße 104, 90478 Nürnberg, Tel.: (0911) 179 3114, E-Mail: joachim.moeller@iab.de

ว Open Access. @ 2018 Joachim Möller, publiziert von De Gruyter. (c) BY-NC-ND Dieses Werk ist lizensiert unter der Creative Commons Attribution-NonCommercial-NoDerivatives 4.0 Lizenz. 
auf die Integration in den ersten Arbeitsmarkt haben. Dabei soll zwar prinzipiell der Anspruch auf einen Übergang in ungeförderte Beschäftigung nicht aufgegeben werden, vordringlich verfolgt der Soziale Arbeitsmarkt jedoch eine primär sozialpolitisch motivierte Zielsetzung. Sein Kernanliegen besteht darin, die vielfältigen Teilhabedefizite zu mildern, die bei einem langfristigen Ausschluss vom Arbeitsmarkt und dem im Regelfall damit einhergehenden Bezug von Grundsicherungsleistungen zu beobachten sind (vgl. für einen Überblick Ramos Lobato et al. [Hg.], 2016). Der Soziale Arbeitsmarkt setzt also auf die vielfältigen Inklusionseffekte regulärer Erwerbsarbeit durch eine massive Senkung der Arbeitskosten zur Förderung der Erwerbstätigkeit besonders vulnerabler Gruppen.

In den Jahren 2007 bis 2012 existierte mit dem Beschäftigungszuschuss ein Instrument, das darauf abzielte, die soziale Teilhabe der Geförderten zu verbessern und damit der Kernidee eines Sozialen Arbeitsmarkts nahe kam. Für die derzeit im SGB II vorhandenen Varianten öffentlich geförderter Beschäftigung gilt dies hingegen nur sehr bedingt. Um diese Lücke zu schließen, haben sich CDU, CSU und SPD im Koalitionsvertrag darauf verständigt, ein neues Regelinstrument für besonders arbeitsmarktferne Langzeitarbeitslose zu schaffen. Dafür wurden Finanzmittel in Höhe von bis zu vier Milliarden Euro bereitgestellt. Konkret sieht der mittlerweile vorliegende Referentenentwurf für bis $\mathrm{zu} 150.000$ langfristig erfolglos integrierter Personen die hohe Förderung sozialversicherungspflichtiger Arbeitsverhältnisse bei privatwirtschaftlichen wie gemeinnützigen Arbeitgebern vor. Die Förderung zur „Teilhabe am Arbeitsmarkt“, so der Name des neuen, im vorgeschlagenen $\S 16 \mathrm{i}$ SGB II geregelten Instruments, ist auf einen Zeitraum von bis zu fünf Jahren angelegt.

Die geplanten Maßnahmen sind vor dem Hintergrund der Entwicklung der Langzeitarbeitslosigkeit von Grundsicherungsempfängern zu sehen. Während diese zwischen 2007 und 2011 von 1,4 Millionen auf rund 920 Tausend stark gesunken ist, ist sie bis zum Jahr 2017 nur noch um etwas mehr als 110 Tausend Personen zurückgegangen. Das geplante Förderinstrumentarium ermöglicht es den Jobcentern, bei einem Teil der Betroffenen, die nachteiligen Folgen des langfristigen Arbeitsmarktausschlusses zu lindern. Dass es grundsätzlich gelingen kann, die wahrgenommene soziale Teilhabe der Geförderten zu verbessern, belegen die Evaluation des Beschäftigungszuschusses (ISG et al., 2011) ebenso wie Studien zu verschiedenen Modellprojekten auf Bundes- wie auf Landesebene (Bauer et al., 2016; Fuchs/Hamann, 2017; IAQ et al., 2018). Aus den Untersuchungen folgt aber auch, dass für den Erfolg des Instruments bestimmte Bedingungen erfüllt sein sollten. Wichtige Befunde fassen wir in Abschnitt 2 zusammen und leiten daraus in Abschnitt 3 Folgerungen für eine zielführende Ausgestaltung des Sozialen Arbeitsmarkts ab. Auf dieser Grundlage diskutieren wir die Regelungen des Referentenentwurfs in Abschnitt 4. 


\section{Zur Wirkung öffentlich geförderter Beschäftigung}

Die öffentliche Beschäftigungsförderung ist ein klassisches Instrument aktiver Arbeitsmarktpolitik. Unter diese Oberkategorie fallen eine Vielzahl einzelner Instrumente, mit denen (sozialversicherungspflichtige) Beschäftigungsverhältnisse teils bei gemeinnützigen Arbeitgebern, teils bei erwerbswirtschaftlich orientierten Betrieben gefördert werden (vgl. Matysik et al., 2011). Im Bereich der Grundsicherung gehören zu dieser Gruppe arbeitsmarktpolitischer Maßnahmen - nach der Abschaffung der Arbeitsbeschaffungsmaßnahmen (ABM) im Jahr 2009 und des Beschäftigungszuschusses im Jahr 2012 - derzeit die Arbeitsgelegenheiten sowie die Förderung von Arbeitsverhältnissen. Jenseits der Regelförderung existiert seit 2015 zudem das bis Ende 2018 befristete Bundesprogramm „Soziale Teilhabe am Arbeitsmarkt“.

Die verschiedenen Instrumente der Beschäftigungsförderung richten sich an Arbeitslose mit besonders geringen Arbeitsmarktchancen. Für sie sind häufig Maßnahmen nicht geeignet, die wie die Förderung beruflicher Weiterbildung auf eine unmittelbare Integration in den ersten Arbeitsmarkt zielen. Stattdessen soll die geförderte Beschäftigung zunächst die grundlegende Beschäftigungsfähigkeit der Teilnehmerinnen und Teilnehmer verbessern und dadurch mittelfristig ihre Arbeitsmarktchancen erhöhen. Gleichzeitig folgt die Beschäftigungsförderung dem Leitgedanken, Arbeit statt Arbeitslosigkeit zu finanzieren und beabsichtigt daher - wenn auch vielfach nur als Nebeneffekt - die mit dem Ausschluss vom Arbeitsmarkt einhergehenden Teilhabedefizite zu lindern. Weitere Zielsetzungen des Einsatzes geförderter Beschäftigung können die Überprüfung der Arbeitsbereitschaft von Arbeitslosen oder die Entlastung regionaler Arbeitsmärkte sein, die eine sehr hohe Arbeitslosigkeit aufweisen.

Die vorliegenden Studien zu den Varianten öffentlich geförderter Beschäftigung, die entsprechend dem Konzept eines Sozialen Arbeitsmarkts sozialversicherungspflichtige Arbeitsverhältnisse fördern, verweisen jedoch neben positiven Effekten zugleich auf unbeabsichtigte Nebenwirkungen. So ist ein hervorzuhebendes Ergebnis dieser Untersuchungen, dass der Einsatz geförderter Beschäftigung teilweise mit einem hohen Lock-in-Effekt verbunden ist (vgl. für einen Überblick Wolff/Stephan, 2013). Dieser Effekt ist vor allem darauf zurückzuführen, dass den Geförderten durch die (teils in Vollzeit) ausgeübte Tätigkeit weniger Zeit für die Arbeitsuche zur Verfügung steht als während der Arbeitslosigkeit. Zugleich ist für geförderte Personen der monetäre Anreiz, eine ungeförderte Beschäftigung zu suchen, geringer als für Arbeitslose. 
Sehr hohe Lock-in-Effekte wurden für Teilnahmen an Arbeitsbeschaffungsmaßnahmen (ABM) kurz nach der Jahrtausendwende nachgewiesen. Den Befunden von Caliendo et al. (2004) zufolge war die Wahrscheinlichkeit, einer ungeförderten versicherungspflichtigen Beschäftigung nachzugehen, für Geförderte in Westdeutschland während des Teilnahmezeitraumes gegenüber einer Vergleichsgruppe um bis zu 20 Prozentpunkte verringert. Ein Grund dafür ist darin zu sehen, dass damals auch viele arbeitsmarktnahe Personen gefördert wurden. Für die Zeit nach den Hartz-Reformen ergeben sich andere Befunde. So zeigt die Studie von Hohmeyer/Wolff (2010) für an ABM und Arbeitsgelegenheiten in der Entgeltvariante teilnehmenden Bezieherinnen und Bezieher von Arbeitslosengeld (ALG) II im Jahr 2005, dass die Lock-in-Effekte gering ausfallen. Für viele der untersuchten Gruppen wurden bereits im ersten oder spätestens im zweiten Jahr nach Förderbeginn recht positive Eingliederungswirkungen erzielt. Das dürfte auch damit zusammenhängen, dass in der Folge der Hartz-Reformen die Teilnahmedauer kürzer ausfiel und stärkere Anreize zur Aufnahme einer ungeförderten Erwerbstätigkeit geschaffen wurden.

Für den Beschäftigungszuschuss wurden ebenfalls nicht unerhebliche Lockin-Effekte festgestellt (ISG et al., 2011). So reduzierte sich durch die Teilnahme an einer solchen Maßnahme die Wahrscheinlichkeit der Aufnahme einer ungeförderten Beschäftigung um bis zu 10 Prozentpunkte. Ausschlaggebend hierfür war mutmaßlich die lange Förderdauer, die für Arbeitslose und für Arbeitgeber gleichermaßen attraktiv gewesen sein dürften. Diese betrug zunächst bis zu zwei Jahre, konnte anschließend unter gewissen Voraussetzungen aber auf unbestimmte Dauer fortgesetzt werden.

Gleichzeitig konnte die Evaluation des Beschäftigungszuschusses jedoch zeigen, dass das Instrument die wahrgenommene soziale Teilhabe der Geförderten signifikant verbesserte. Der begleitend durchgeführten qualitativen Befragung zufolge ist die Teilhabewirkung der Förderung entscheidend auf die wahrgenommene „Normalisierung“ der eigenen Lebensumstände zurückzuführen. Begünstigt wurde diese Wahrnehmung wesentlich von vier Bedingungen: der Förderung sozialversicherungspflichtiger Arbeitsverhältnisse, dem damit zusammenhängenden Gefühl, mit dem Arbeitslohn über selbstverdientes Geld zu verfügen, der Überwindung des Leistungsbezugs sowie der vergleichsweise langen Förderdauer (Hirseland et al., 2012; Ramos Lobato, 2017). Die teilhabesteigernde Wirkungen öffentlich geförderter Beschäftigung haben auch die Untersuchungen der Modellprojekte öffentlich geförderte Beschäftigung in Nordrhein-Westfalen (Bauer et al., 2016) sowie des Bundesprogramms „Teilhabe am Arbeitsmarkt“ (IAQ et al., 2018) bestätigt. 


\section{Ausgestaltung eines Sozialen Arbeitsmarkts}

Die genaue Einhaltung der auf besonders arbeitsmarktferne Langzeitarbeitslose beschränkten Zielgruppe ist eine entscheidende Erfolgsbedingung des Sozialen Arbeitsmarkts. Im Auge zu behalten ist ein inhärenter Zielkonflikt: Mit Blick auf die intendierte Stärkung sozialer Teilhabechancen und aus dem Bestreben heraus, den negativen gesundheitlichen und psychischen Folgen der Arbeitslosigkeit entgegenzuwirken, erscheint einerseits eine längere Förderdauer als bei anderen Instrumenten sinnvoll. Andererseits entsteht möglicherweise gerade dadurch ein ausgeprägter Lock-in-Effekt. Koch/Kupka (2012) zufolge erweist sich dieser Zielkonflikt als umso gravierender, je weniger die Zuweisungskriterien eingehalten werden.

Die Forderung einer strikten Konzentration auf besonders arbeitsmarktferne Arbeitslose hat Konsequenzen für die Ausgestaltung der Beschäftigungsverhältnisse. Die Zielgruppe ist durch Häufung von gravierenden Vermittlungshemmnissen gekennzeichnet (Beste/Trappmann, 2016). Dazu gehören beispielsweise gesundheitliche und psychische Probleme. Es ist also davon auszugehen, dass ein beträchtlicher Teil des avisierten Personenkreises nicht in der Lage sein wird, in Vollzeit zu arbeiten. Die Ausgestaltung der Arbeitszeit sollte der individuellen Leistungsfähigkeit in dieser Hinsicht Rechnung tragen.

Ein weiterer Erfolgsfaktor im Sinne der Zielsetzung des Instruments ist, dass die Aufnahme einer Tätigkeit im Sozialen Arbeitsmarkt auf Freiwilligkeit basiert. Eine (sanktionsbewehrte) Verpflichtung wäre bei einer Maßnahme widersinnig, die primär die soziale Teilhabe verbessern soll. Da zudem davon auszugehen ist, dass für einen Teil der Zielgruppe nach langer Arbeitslosigkeit bei u. U. schlechter gesundheitlicher oder psychischer Verfassung die Aufnahme einer Beschäftigung eine Herausforderung darstellt und Selbstvertrauen erfordert, könnte Druck oder gar eine Sanktionierung seitens des Jobcenters sogar kontraproduktiv wirken.

Die Arbeitsanforderungen müssen der zumeist eingeschränkten Leistungsfähigkeit der Geförderten Rechnung tragen. Andernfalls kann es zu einer Überforderung der Beschäftigten kommen, wodurch die Gefahr eines vorzeitigen Abbruchs der Maßnahme steigt. Ein Sozialer Arbeitsmarkt, der keine Betreuung gesundheitlich beeinträchtigter Personen, psychisch Kranker oder allgemein jener Menschen, die bislang am Rande der (Arbeits-)Gesellschaft standen, vorsieht, würde zweifellos sein Ziel verfehlen. Deswegen ist eine sozialpädagogische Begleitung der Geförderten, wie sie die oben bereits erwähnten Modellprojekte in Nordrhein-Westfalen vorsahen, zwingend erforderlich. Je nach individueller Problemlage ist deren Ansiedlung im Einsatzbetrieb selbst oder aber bei einem externen Träger sinnvoller (Bauer/Kupka, 2016).

Den vorliegenden Untersuchungen zufolge sind Teilhabeeffekte dann besonders stark ausgeprägt, wenn die geförderte Beschäftigung einem regulären 
Arbeitsverhältnis möglichst ähnlich ist. Dies schließt neben einer sinnstiftenden Aufgabe auch eine auskömmliche Vergütung ein, die zumindest für Alleinstehende in Vollzeit auch einen Abstand zum Grundsicherungsniveau aufweist.

Bei der Festsetzung der Höhe der Vergütung sind allerdings ganz verschiedene Aspekte $\mathrm{zu}$ beachten. $\mathrm{Zu}$ berücksichtigen ist, dass der geförderte Personenkreis eine eher geringe Produktivität aufweisen dürfte und zudem einen Betreuungsbedarf (betriebsinternes oder externes Coaching) erwarten lässt. Diese Betreuung stellt aber auch einen Kostenfaktor da, der in der Gesamtschau beachtet werden muss. Weiterhin sind in Bezug auf die Höhe der Vergütung die Auswirkungen auf ungeförderte Beschäftigte zu bedenken, die möglicherweise im selben Betrieb arbeiten. Zum einen könnte hier ein fehlender Abstand zu der eigenen Vergütung als ungerecht empfunden werden, zum anderen eine zu geringe Bezahlung als Unterbietungswettbewerb betrachtet werden.

In der Abwägung der verschiedenen Aspekte wäre eine Bezahlung unterhalb des Mindestlohns nicht zu empfehlen. Um Beschäftigung auch in tarifgebundenen Unternehmen zu ermöglichen, käme auch in diesen Fällen eine Bezahlung in Betracht, die der untersten Tarifgruppe entspricht. In jedem Fall ist aber in diesem Zusammenhang zu bedenken, dass die geförderten Arbeitsverhältnisse bereits aufgrund der langen Förderdauern auch für vergleichsweise arbeitsmarktnahe erwerbsfähige Leistungsberechtigte attraktiv sein könnten. Ein Creaming bei der Auswahl der Geförderten würde aber den Erfolg des Sozialen Arbeitsmarkts grundsätzlich gefährden.

Anstelle einer unbefristeten Dauer, wie sie beim Beschäftigungszuschuss möglich war, sollte die Förderung in längere Abschnitte unterteilt, an deren Ende jeweils die Fördervoraussetzungen überprüft werden. Bei einer unveränderten Prognose der Erwerbschancen könnte sich dann ein neuer Abschnitt anschließen (Kupka/Wolff, 2013). Bei einer von vornherein unbefristeten Dauer wird die Möglichkeit ausgeschlossen, dass eine Person in der geförderte Beschäftigung wieder Kompetenzen entwickelt und Qualifikationen erwirbt, die einen Übergang in den ersten Arbeitsmarkt erreichbar erscheinen lassen. Der Soziale Arbeitsmarkt muss im Umfang begrenzt sein. Deshalb ist auch eine gewisse Fluktuation erforderlich. Wenn Geförderte nicht oder nur in geringem Umfang die Maßnahme verlassen, könnten andere anspruchsberechtigte Personen leer ausgehen. Dies würde auch die arbeitsmarktpolitische Handlungsfähigkeit der Jobcenter beeinträchtigen. 


\section{Bewertung}

Langzeitarbeitslosigkeit führt zum Verlust von Kompetenzen und Kenntnissen (Dequalifikation), zu Frustration bei den Betroffenen (Demotivation) und gefährdet letztlich den Zusammenhalt in einer Gesellschaft (Desintegration). Deswegen gibt es sehr gute Gründe, sie zu bekämpfen. Dies gilt in besonderem Maße auch für die Langzeitarbeitslosigkeit der Grundsicherungsempfängerinnen und -empfänger. Trotz insgesamt guter Arbeitsmarktlage hat sich ein Teil der Langzeitarbeitslosigkeit in Deutschland verfestigt. Bei einem Teil der betroffenen Personen lässt die Kumulation integrationshemmender Merkmale - die sich zum Teil auch erst durch die längerfristige Erwerbslosigkeit eingestellt hat - die Einmündung in den ersten Arbeitsmarkt faktisch als unrealistisch erscheinen.

Die Einrichtung eines sozialen Arbeitsmarktes erscheint vor diesem Hintergrund dringend erforderlich. Allerdings dürfen die Risiken nicht übersehen werden. Ein sozialer Arbeitsmarkt muss ultima ratio sein. Entscheidend ist es, bestmöglich sicherzustellen, dass tatsächlich nur ein Personenkreis gefördert wird, der ansonsten nahezu keine Beschäftigungschance hätte. Fehlzuweisungen wären fatal, da sie nicht nur öffentliche Mittel, sondern auch persönliche Ressourcen vergeuden. Die Sorgfalt bei der Auswahl der Personen entscheidet somit über den Erfolg des Instruments.

Da sich im Übrigen die Beschäftigungsfähigkeit und -chancen von Personen im Zeitablauf durchaus verändern können, wäre eine Weiterförderung turnusmäßig zu überprüfen. Dabei sollten allerdings die Abstände nicht zu kurz gewählt werden, da auch ein Sicherheitsgefühl der betroffenen Personen zum wahrgenommenen Teilhabeerfolg beiträgt. Anstelle einer Beschränkung des Sozialen Arbeitsmarktes auf einen Zeitraum von fünf Jahren ohne Verlängerung würden wir im Sinne der Vorschläge von Kupka/Wolff (2013) eine Verlängerung der Beschäftigung nach regelmäßiger Prüfung Z. B. in einem Zwei-Jahres-Rhythmus vorziehen.

Zweifellos darf man bei der Einrichtung eines Sozialen Arbeitsmarktes nicht darüber hinwegtäuschen, dass es sich dabei nicht um eine nach engen Kriterien wirtschaftliche Maßnahme handelt, bei denen die Kosten des Instruments gegen zusätzliche Integrationen in den Arbeitsmarkt aufgerechnet werden können. Hier stehen die intangiblen Vorteile einer Investition in gesellschaftlichen Zusammenhalt im Vordergrund.

$\mathrm{Zu}$ warnen ist vor zu großen Erwartungen. Es gilt zu bedenken, dass zumindest ein Teil der leistungsberechtigten extrem Langzeitarbeitslosen des SGB II aufgrund erheblicher gesundheitlicher Einschränkungen und anderer Faktoren den Anforderungen einer geförderter Beschäftigung in Vollzeit nicht gewachsen sein dürfte. Hier wäre ein behutsamer Einstieg empfehlenswert. Die vorge- 
sehene sozialpädagogische Betreuung im Rahmen eines Jobcoachings ist dabei sehr zu begrüßen, da sie die Beschäftigung gerade in der Anfangsphase stabilisieren kann (Bauer/Fertig/Fuchs, 2016). Auch bei den Zugangszahlen sollte man zunächst mit einer überschaubaren Größenordnung beginnen. Dabei gilt es nicht nur die Übergangsprobleme, sondern auch die Zuweisung genau zu beobachten, um Creaming-Effekte und ähnliche Probleme, wie sie sich beim Beschäftigungszuschuss gezeigt haben, zu vermeiden.

Der vorliegende Gesetzentwurf baut insofern einer Fehlzuweisung vor, als er die Zugangskriterien mit einem sechsjährigen Leistungsbezug mit nur geringer Erwerbsbeteiligung in einem Zeitraum von sieben Jahren sehr restriktiv festlegt. Damit ist weitgehend sichergestellt, dass es sich um eine sehr arbeitsmarktferne Klientel handelt, die als Zielgruppe in Betracht kommt. Gleichwohl können mit dieser Definition auch Personen teilnahmeberechtigt werden, die durchaus Chancen auf reguläre Beschäftigung haben. Man denke etwa an Alleinerziehende mit einem Berufsabschluss. Zusätzlich wünschenswert wäre daher die Aufnahme eines Passus, dass nach Einschätzung der Jobcenter eine Erwerbstätigkeit auf dem ersten Arbeitsmarkt voraussichtlich innerhalb der nächsten 24 oder 36 Monate ohne die Förderung aussichtslos ist. Sinnvoll wäre auch eine Altersuntergrenze, da bei jungen Erwachsenen vorrangig Qualifizierungsinstrumente eingesetzt werden sollten. Umgekehrt wäre auch eine Härtefallregelung überlegenswert, die den Zugang zum Sozialen Arbeitsmarkt unabhängig von der bisherigen Länge der Arbeitslosigkeit ermöglicht, wenn ein besonderes gravierendes Ereignis eingetreten ist, das mit hoher Sicherheit zum langfristigen Ausschluss vom regulären Arbeitsmarkt führt. Betroffene Personen müssten u. U. ansonsten sehr lang auf die Zugangsberechtigung warten.

Die degressive Ausgestaltung des Zuschusses unterstellt die Aussicht auf eine positive Entwicklung der Produktivität bzw. Beschäftigungsfähigkeit bei den Geförderten. Hier besteht ein gewisses Risiko, das potenzielle Arbeitgeber doch nach Leistungsfähigkeit innerhalb der Zielgruppe auswählen, sodass wiederum Creaming-Effekte nicht ganz ausgeschlossen werden können.

Konsequent erscheint es, dass beim neuen $\S 16$ i SGB II keine Arbeitslosenversicherungspflicht vorgesehen ist. Ansonsten bestünde die Gefahr, dass es zu den bekannten Drehtüreffekten kommt, bei der die Betroffenen zwischen Leistungsbezug und geförderter Beschäftigung verschoben werden.

Praktische Umsetzungsprobleme bei der Einführung des Sozialen Arbeitsmarkts liegen auch darin begründet, dass zum einen die Trägerstrukturen im gemeinnützigen oder öffentlichen Bereich möglicherweise nicht ausreichend vorhanden sind. Bei staatlichen, kommunalen oder gemeinnützigen Trägern wird es darauf ankommen, die Kriterien der Zusätzlichkeit und Wettbewerbsneutralität mit Augenmaß zu bewerten. Zum anderen ist durchaus fraglich, ob die 
Einstellung extrem langfristig Arbeitsloser für privatwirtschaftliche Unternehmen selbst dann ausreichend attraktiv ist, wenn ein Großteil der Arbeitskosten übernommen wird. Hinweise darauf, dass eine Senkung der Arbeitskosten in der privaten Wirtschaft nicht zu Mehreinstellungen von arbeitsmarktfernen Personen führt, ergeben sich Z. B. aus der Tatsache, dass die Ausnahmeregelungen im Mindestlohngesetz bei der Einstellung von Langzeitarbeitslosen kaum in Anspruch genommen worden sind (vom Berge et al., 2016). Hier wäre $\mathrm{zu}$ fragen, ob die Bereitschaft zur Mitwirkung privatwirtschaftlicher Unternehmen bei der Umsetzung des Sozialen Arbeitsmarktes nicht auch durch nicht-pekuniäre Anreize erhöht werden könnte. Beispielsweise könnte bei entsprechender Kooperation etwa ein Label „Den sozialen Arbeitsmarkt unterstützendes Unternehmen“ vergeben werden, das im Arbeitgeber-Branding verwendet werden könnte.

\section{Literatur}

Bauer, Frank, Michael Fertig und Philipp Fuchs (2016), „Modellprojekte öffentlich geförderte Beschäftigung in NRW“: Teilnehmerauswahl und professionelle Begleitung machen den nterschied. IAB-Kurzbericht, 10/2016.

Bauer, Frank und Peter Kupka (2016), „Öffentlich geförderte Beschäftigung für Langzeitarbeitslose - die Bedeutung sozialpädagogischer Betreuung“. In: Archiv für Wissenschaft und Praxis der sozialen Arbeit, Jg. 47, H. 4, S. 74-83.

Beste, Jonas und Mark Trappmann (2016), „Erwerbsbedingte Abgänge aus der Grundsicherung: Der Abbau von Hemmnissen macht's möglich“, IAB-Kurzbericht, 21/2016, Nürnberg.

Caliendo, Marco, Reinhard Hujer und Stephan L. Thomsen (2004), „Evaluation der Eingliederungseffekte von Arbeitsbeschaffungsmaßnahmen in reguläre Beschäftigung für Teilnehmer in Deutschland“. In: Zeitschrift für ArbeitsmarktForschung, Jg. 37, H. 3, S. 211-237.

Fuchs, Philipp und Silke Hamann (2017), „Öffentlich geförderte Beschäftigung von Langzeitarbeitslosen. Betreuung, Wohlfahrtseffekte und Beschäftigungsperspektiven bei privaten und gemeinnützigen Arbeitgebern. In: Sozialer Fortschritt, Jg. 66, H. 9, S. 611-631.

Hirseland, Andreas, Philipp Ramos Lobato und Tobias Ritter (2012), „Soziale Teilhabe durch geförderte Beschäftigung? Das Beispiel des Beschäftigungszuschusses“. In: WSI-Mitteilungen, Jg. 65, H. 2, S. 94-102.

Hohmeyer, Katrin und Joachim Wolff (2010), „Direct job creation in Germany revisited: Is it effective for welfare recipients and does it matter whether participants receive a wage?“ IAB-Discussion Paper, 21/2010.

IAQ, ZEW, Zoom, SOKO (2018), Evaluation des Bundesprogramms „Soziale Teilhabe am Arbeitsmarkt“ (Zb1-04812-2/31) - Zweiter Zwischenbericht -, Bundesministerium für Arbeit und Soziales Forschungsbericht 504.

ISG; IAB; RWI (2011), Endbericht der Evaluation der Leistungen zur Beschäftigungsförderung nach $\S 16$ e Absatz 10 des Zweiten Buches Sozialgesetzbuch, Bundestagsdrucksache $17 / 6880$. 
Koch, Susanne; Kupka, Peter (2012): Öffentlich geförderte Beschäftigung. Integration und Teilhabe für Langzeitarbeitslose. WISO Diskurs, Bonn.

Kupka, Peter und Joachim Wolff (2013), „Sozialer Arbeitsmarkt: Ein Gerüst, das gesellschaftliche Teilhabe ermöglicht?“ In: IAB-Forum, Nr. 2, S. 70-75.

Matysik, Alexander, Peer Rosenthal und Peer Sommer (2011), „Öffentlich geförderte sozialversicherungspflichtige Beschäftigung in Deutschland. Aktuelle Instrumente, Programme und Konzepte“, WISO Diskurs, August 2011.

Ramos Lobato, Philipp (2017), Geförderte Beschäftigung für Langzeitarbeitslose. Integrationserleben am Rande der Arbeitsgesellschaft. Wiesbaden.

Ramos Lobato, Philipp (Hrsg.); Promberger, Markus (Hrsg.); Christoph, Bernhard (Hrsg.); Lietzmann, Torsten (Hrsg.) (2016), „Krisenerfahrung Hartz IV: Lebenssituationen im Grundsicherungsbezug“. In: WSI-Mitteilungen, Jg. 69, H. 5, S. 324-392.

Vom Berge, Philipp, Isabell Klingert, Sebastian Becker, Julia Lenhart, Simon Trenkle und Matthias Umkehrer (2016), Mindestlohnausnahme für Langzeitarbeitslose: Wenig wirksam und kaum genutzt“. IAB-Kurzbericht, 23/2016, Nürnberg.

Wolff, Joachim und Gesine Stephan (2013), „Subsidized work before and after the German Hartz reforms. Design of major schemes, evaluation results and lessons learnt“. In: IZA Journal of Labor Policy, Vol. 2. 